\title{
1 Einführung zum Hintergrund, Inhalt und Aufbau der Arbeit
}

Medien bestimmen den Lebensalltag von Kindern und Jugendlichen im Deutschland des 21. Jahrhunderts in entscheidender Weise. Neben der Familie, der Schule und den gleichaltrigen Freunden verbringen Kinder und Jugendliche deutlich mehr Zeit mit - vor allem elektronischen - Medien, als mit anderen Freizeittätigkeiten. Vor diesem Hintergrund erscheint es plausibel, dass beispielsweise Charlton und Neumann-Braun (1992) bereits Anfang der 1990er-Jahre von einer Medienkindheit sprachen. Charlton schränkt diese Bezeichnung zugleich mit dem Hinweis ein, diese Bezeichnung sei nicht mit der Feststellung gleichzusetzen, dass Kinder wirklich mehr Zeit mit Medien verbringen als mit wichtigen Bezugspersonen (Freunde oder Familie). Es sei vielmehr beabsichtigt, mit diesem Begriff das Aufwachsen von Kindern in der heutigen Gesellschaft von den Bedingungen abzugrenzen, unter denen Kinder Mitte des 20. Jahrhunderts groß geworden sind (Charlton, 2004, S. 144). Doch auch diese Einschränkungen zur Bedeutung des Begriffes Medienkindheit bedürfen aktuell einer Revision.

Die in den 1990er-Jahren sehr auf das Fernsehen fokussierte Diskussion über den Stellenwert von Medien im Leben von Kindern muss tatsächlich neu geführt werden, da digitale, interaktive Medien inzwischen im „Mainstream“ der Gesellschaft angekommen und im Lebensalltag aller, gerade dem der Kinder und Jugendlichen, eine maßgebliche Rolle spielen. Stellten Kübler und Swoboda noch 1998 fest, dass das Fernsehen als das damalige Leitmedium unter Kindern und Jugendlichen im Alltag von Vorschulkindern keine hervorgehobene Rolle spielte und somit von einer "Fernsehkindheit" nicht eigentlich gesprochen werden konnte (Kübler, H. D. \& Swoboda, 1998), so sind neben die Fernsehnutzung und die Nutzung von Hör- und Printmedien inzwischen weitere Mediennutzungsformen getreten, die nicht nur mit traditionellen Medien konkurrieren, sondern auch Einfluss auf andere Lebensbereiche der Kinder nehmen.

Die Frage nach den Auswirkungen einer zunehmend medial geprägten Lebenswelt auf Kinder und Jugendliche ist vor diesem Hintergrund schnell gestellt. Eng verknüpft ist sie mit zwei weiteren Fragen: Welche Funktionen erfüllen Medien eigentlich im Alltag von Kindern und Jugendlichen? Und wie kann es gelingen, Kindern Kompetenzen in der Nutzung ihrer Medien zu vermitteln, so dass Mediennutzung ein wirklich funktionaler Bestandteil ihres Alltags wird, der ihr Leben bereichert, ohne wichtige entwicklungsförderliche nichtmediale Erfahrungen zu verdrängen? 
Im Jahr 2004 bewilligte die Volkswagenstiftung einem interdisziplinären Forschungsteam unter Federführung des Kriminologischen Forschungsinstituts Niedersachsen (KFN) ein Forschungsprojekt, das diesen Fragen nachgehen sollte. Neben einem neurobiologischen Forschungsteil, in dessen Rahmen hirnphysiologische Prozesse bei der Nutzung von Mediengewaltinhalten durch ein Forschungsteam der Otto-von-Guericke-Universität Magdeburg untersucht wurden, bestand das Forschungsprojekt Mediennutzung und Schulleistung aus drei sozialwissenschaftlichen Studienteilen, die von Mitarbeitern des Kriminologischen Forschungsinstituts Niedersachen durchgeführt wurden.

Im ersten Studienteil, einer Befragung von 5.529 westdeutschen Viertklässlern und 14.301 westdeutschen Neuntklässlern ${ }^{1}$, wurden korrelative Zusammenhänge zwischen Mediennutzung, Schulleistung und Sozialverhalten im Grundund Sekundarschulalter untersucht, die im Rahmen dieser Arbeit insbesondere in den Kapiteln 2 und 4 noch näher dargestellt werden (vgl. auch Mößle et al., 2007). Dieser erste sozialwissenschaftliche Studienteil wurde neben der Volkswagenstiftung vom Programm Polizeiliche Kriminalprävention der Länder und des Bundes (ProPK), den Landesmedienanstalten der Länder NordrheinWestfalen und Niedersachsen sowie den beteiligten Kommunen und Landkreisen finanziell gefördert. Mit dem Landkreis Soltau-Fallingbostel und der Stadt Oldenburg, zwei der Regionen, in denen im Jahr 2005 Schülerbefragungen stattfanden, wurde vereinbart, dass eine erste Stufe des Präventionskonzepts in diesen beiden Regionen durchgeführt und getestet werden soll (vgl. auch S. 258 264 in dieser Arbeit).

Im zweiten Studienteil, einem medienpsychologischen Experiment zum Einfluss der Gewaltmediennutzung auf die Lern- und Konzentrationsleistungen junger Erwachsener (vgl. Rehbein, 2010), wurden die genauen Hintergründe des schulleistungsmindernden Einflusses rezeptiver und interaktiver Gewaltmediennutzung untersucht, die in der Literatur erwähnt und in der Schülerbefragung 2005 erneut gefunden wurden.

Die vorliegende Arbeit entstand im Rahmen des dritten sozialwissenschaftlichen Studienteils des Projekts Mediennutzung und Schulleistung. In diesem dritten Studienteil mit dem Namen Berliner Längsschnitt Medien wurden Berliner Grundschülerinnen und Grundschüler über vier Jahre lang begleitet und wiederholt untersucht. Für eine Teilgruppe dieser Schülerinnen und Schüler

\footnotetext{
1 Zur Methode und Stichprobenziehung vgl. die Beschreibung der KFNGrundschulbefragung 2005 auf S.29 und die Beschreibung der KFNNeuntklässlerbefragung 2005 bei Mößle, Kleimann und Rehbein (2007, S. 48-54)
} 
wurde ein Medienunterrichtskonzept - Medienlotsen-Unterrichtsprogramm - zur Prävention problematischer Mediennutzungsmuster entwickelt.

Die Darstellung der theoretischen Herleitung und Entwicklung dieser Unterrichtseinheit bildet den Hauptteil der vorliegenden Arbeit. In einem zweiten, empirischen Teil werden Implementation und Effekte des MedienlotsenUnterrichtsprogramms im Rahmen des Berliner Pilotprojektes evaluiert. Dabei wird im Rahmen der Implementationsevaluation auch gezeigt werden, welche Chancen und Probleme sich bei der Einbindung medienerzieherischer Inhalte in den schulischen Unterricht ergeben. Die Effektevaluation zeigt, welche konkreten Effekte mithilfe schulischer Medienerziehung tatsächlich erreicht werden konnten und welche Implikationen die gefundenen Effekte für die Diskussion über schulische Medienerziehung sowie die Debatte über problematische Medienwirkungen und deren Prävention haben.

Breiter Raum wird in dieser Arbeit der Aufarbeitung zentraler Befunde der Medienwirkungsforschung sowie der Ableitung konkreter Anforderungen für schulische Medienerziehungskonzepte gegeben. Dies geschieht vor dem Hintergrund eines großen Mangels theoretisch und empirisch fundierter Konzepte schulischer Medienerziehung. So existieren zwar mehr als hundert Definitionen von Medienkompetenz (vgl. S. 159 in dieser Arbeit), jedoch beziehen sich bisherige Konzepte (kindlicher) Medienkompetenz kaum auf Ergebnisse der empirischen Medienwirkungsforschung und wurden bisher nur sehr rudimentär empirisch überprüft. Zwar wurden insbesondere in den USA bereits Konzepte zur schulischen Prävention problematischer Mediennutzung auf der Grundlage der empirischen Medienwirkungsforschung entwickelt und auch evaluiert, elaborierte theoretische Überlegungen zur inhaltlichen und didaktischen Konzeption dieser Projekte finden sich hingegen allenfalls ansatzweise. Diese Theorielücken sollen im Rahmen dieser Arbeit geschlossen werden.

Nachfolgend wird die Untergliederung dieser Arbeit nach den einzelnen Kapiteln dargestellt:

Im Anschluss an diese Einführung wird im zweiten Kapitel gezeigt, welch breiten Raum die Nutzung elektronischer Medien bereits im Alltag von Kleinund Vorschulkindern einnimmt und wie elektronische Mediennutzung in den Alltag von Grundschulkindern und Jugendlichen integriert ist. Im dritten Kapitel werden die Entwicklungsdeterminanten kindlicher und jugendlicher Medienkompetenz dargestellt und die Funktionen der Mediennutzung für die kindliche Entwicklung erläutert. Im vierten Kapitel werden die wichtigsten aktuellen Diskussionen über mögliche negative Effekte der dysfunktionalen Nutzung elektronischer Medien dargestellt, wobei der Schwerpunkt auf Erkenntnisse zu den Zusammenhängen zwischen elektronischer Mediennutzung und der Schul- 
leistung Minderjähriger, sowie auf Erkenntnisse zum Zusammenhang zwischen elektronischer Mediennutzung und dem Sozialverhalten von Kindern und Jugendlichen gelegt wird.

Nachdem im Rahmen des fünften Kapitels gezeigt wird, dass elterliche Medienerziehung zwar ein geeignetes Instrument zur Vermeidung negativer Mediennutzungseffekte sein kann, diese aber nur unzureichend im Erziehungshandeln vieler Eltern verankert ist, werden im sechsten Kapitel drei Dimensionen problematischer kindlicher und jugendlicher Mediennutzung definiert und quantifiziert: Zeitlich problematische Mediennutzung, inhaltlich problematische Mediennutzung und funktional problematische Mediennutzung. Im siebten Kapitel dieser Arbeit werden mit der Medienkompetenz- Perspektive und der Health-Promotion-Perspektive zwei theoretische Ansätze dargestellt, deren methodisches Instrumentarium auf seine Eignung hinsichtlich der Prävention problematischer Mediennutzung von Minderjährigen überprüft wird. Dabei wird deutlich, dass die Medienkompetenz-Perspektive (als der thematisch zunächst naheliegende Ansatz) aufgrund ihrer Unschärfe, ihrer großen Skepsis bezüglich der Ergebnisse der Medienwirkungsforschung und aufgrund eines nur unzureichend konkret entwickelten Instrumentariums problemzentrierter Medienerziehung bei der Entwicklung eines Präventionsprogramms zur Vermeidung problematischer Mediennutzungsmuster nur wenig hilfreiche Ansätze bietet. Der Health-Promotion-Ansatz, mit dem in dieser Arbeit die Forschung und Praxis gesellschaftlicher Gesundheitsförderung bezeichnet wird, bietet ungleich mehr konkrete Ansatzpunkte, wenngleich sie auf das Feld der problematischen Mediennutzung bisher wenig angewendet wurden. Aus den Erkenntnissen der Health-Promotion-Forschung werden im achten Kapitel Folgerungen für die Entwicklung eines eigenen schulischen Präventionsprogramms zur Vermeidung problematischer Mediennutzungsmuster im Kindesalter entwickelt.

Im neunten Kapitel wird ein Medienlotsen-Unterrichtsprogramm zur schulischen Medienerziehung im Grundschulalter vorgestellt, welches auf den in den Kapiteln eins bis acht dargestellten Grundlagen basiert. Dabei wird auch dargestellt, welche konkreten praktischen Schwierigkeiten sich bei der Implementation dieses Konzeptes im Rahmen seines Einsatzes in Berliner Grundschulklassen ergaben. Im zehnten Kapitel werden auf der Grundlage eines Kontrollgruppenvergleichs die Effekte des Unterrichtskonzeptes auf die Mediennutzung und die schulischen Leistungen der Berliner Schülerinnen und Schüler und die Medienerziehung durch ihre Eltern dargestellt. Hier wird gezeigt, dass das Unterrichtsprogramm insbesondere die Entwicklung funktional problematischer Mediennutzungsmuster signifikant verhindern konnte, während Effekte auf zeitlich oder inhaltlich problematische Mediennutzungsmuster nur 
tendenziell festgestellt werden konnten. Zudem wird gezeigt, dass die Kinder der Unterrichtsklassen im Projektverlauf signifikant bessere Mathematiknoten aufwiesen als Kinder der Kontrollgruppe.

Im elften Kapitel werden die Ergebnisse der Konzeptentwicklung und der Evaluation der Unterrichtseinheit im Kontext der in den Kapiteln zwei bis fünf dargestellten bisherigen Forschungsbefunde diskutiert. Hier wird insbesondere dargestellt, welche neuen Impulse die vorliegende Arbeit für die Definition problematischer Mediennutzungsmuster gegeben hat, in welche Richtung neue Modelle zum Zusammenhang zwischen Mediennutzung und Schulleistung weiterentwickelt werden sollten und was die Ergebnisse der Unterrichtsevaluation für die schulische Medienerziehung in Deutschland bedeuten. 
